\title{
Exponential Attractors of the Nonclassical Diffusion Equations with Lower Regular Forcing Term
}

\section{Yanjun Zhang, Qiaozhen Ma*}

School of Mathematics and Statistics, Northwest Normal University, Lanzhou, China Email: 2003xbsd@163.com, ${ }^{*}$ maqzh@nwnu.edu.cn

Received 19 January 2014; revised 19 February 2014; accepted 25 February 2014

Copyright (C) 2014 by authors and Scientific Research Publishing Inc.

This work is licensed under the Creative Commons Attribution International License (CC BY). http://creativecommons.org/licenses/by/4.0/

(c) (i) Open Access

\section{Abstract}

In this article, we prove the existence of exponential attractors of the nonclassical diffusion equation with critical nonlinearity and lower regular forcing term. As an additional product, we show that the fractal dimension of the global attractors of this problem is finite.

\section{Keywords}

Nonclassical Diffusion Equations; Exponential Attractor; Critical Exponent; Lower Regular Forcing Term

\section{Introduction}

We consider the asymptotic behavior of solutions to be the following nonclassical diffusion equation:

$$
\left\{\begin{array}{l}
u_{t}-\Delta u_{t}-\Delta u+f(u)=g(x), \text { in } \Omega \times \mathbb{R}^{+}, \\
u(x, 0)=u_{0}(x), \\
\left.u\right|_{\partial \Omega}=0,
\end{array}\right.
$$

where $\Omega \subset \mathbb{R}^{N}(N \geq 3)$ is a bounded domain with smooth boundary $\partial \Omega$, and the external forcing term $g(x) \in H^{-1}(\Omega)$, non-linear function $f \in \mathcal{C}^{1}(\mathbb{R})$ with $f(0)=0$ and satisfies the following conditions:

$$
\left|f^{\prime}(s)\right| \leq C_{0}\left(1+|s|^{\frac{N+2}{N-2}-1}\right) \text { for all } s \in \mathbb{R},
$$

"Corresponding author.

How to cite this paper: Zhang, Y.J. and Ma, Q.Z. (2014) Exponential Attractors of the Nonclassical Diffusion Equations with Lower Regular Forcing Term. International Journal of Modern Nonlinear Theory and Application, 3, 15-22. 
and

$$
\liminf _{|s| \rightarrow \infty} \frac{f(s)}{s}>-\lambda_{1}
$$

where $C_{0}$ is a positive constant and $\lambda_{1}$ is the first eigenvalue of $-\Delta$ on $H_{0}^{1}(\Omega)$. The number $\frac{N+2}{N-2}-1$ is called the critical exponent; since the nonlinearity $f$ is not compact in this case, this is one of the essential difficulties in studying the asymptotic behavior.

This equation appears as a nonclassical diffusion equation in fluid mechanics, solid mechanics and heat conduction theory, see for instance [1]-[3] and the references therein.

Since Equation (1.1) contains the term $-\Delta u_{t}$, it is different from the usual reaction diffusion equation essentially. For example, the reaction diffusion equations has some smoothing effect, that is, although the initial data only belongs to a weaker topology space, the solution will belong to a stronger topology space with higher regularity. However, for Equation (1.1), if the initial data $u_{0}$ belongs to $H_{0}^{1}(\Omega)$, the solution $u(x, t)$ with $u(x, 0)=u_{0}$ is always in $H_{0}^{1}(\Omega)$ and has no higher regularity because of $-\Delta u_{t}$, which is similar to the hyperbolic equation. Consequently, its dynamics would be more complex and interesting.

The long-time behavior of the solutions of (1.1) has been considered by many researchers; see, e.g. [4]-[9], and the references therein. For instance, for the case $g(x) \in L^{2}(\Omega)$, the existence of a global attractor of (1.1) in $H_{0}^{1}(\Omega)$ was obtained in [4] under the assumptions that $f$ satisfies (1.2) and (1.3) corresponding to $N=3$ and the additional condition $|f(s)| \leq c\left(1+|s|^{v}\right)(\forall s \in \mathbb{R})$ with $v<5\left(=\left.\frac{N+2}{N-2}\right|_{N=3}\right)$, which essentially requires that the nonlinearity is subcritical. In [7] the authors investigated the existence of the global attractors for $g(x) \in H^{-1}(\Omega)$, and proved the asymptotic regularity and existence of exponential attractors for $g(x, t) \in L_{b}^{2}\left(\mathbb{R} ; L^{2}(\Omega)\right)$ only under the conditions (1.2)-(1.3). Recently, the authors in [9] showed the asymptotic regularity of solutions of Equation (1.1) in $H^{1+\sigma}(\Omega)$ for any $\sigma \in\left[0, \min \left\{1, \frac{N}{2}-1\right\}\right)$ and for $g(x) \in H^{-1}(\Omega), \quad u_{0} \in H_{0}^{1}(\Omega)$ only under the assumptions (1.2)-(1.3).

For the limit of our knowledge, the existence of exponential attractors of Equation (1.1) has not been achieved by predecessors for $g(x) \in H^{-1}(\Omega)$. On the other hand, we note that in [10] the authors scrutinized the asymptotic regularity of the solutions for a semilinear second order evolution equation when $g(x) \in H^{-1}(\Omega)$, and based on this regularity, they constructed a family of finite dimensional exponential attractors. However, they require the following additional technical assumptions besides (1.2) and (1.3):

$$
\left|f^{\prime \prime}(s)\right| \leq C\left(1+\mid s^{\frac{N+2}{N-2}-2}\right) \text {, as } N=3,4,5 ;\left|f^{\prime \prime}(s)\right| \leq C \text {, as } N \geq 6 \text {, for all } s \in \mathbb{R},
$$

and

$$
\left|f^{\prime}(s)\right| \geq C \text {, for all } s \in \mathbb{R} \text {. }
$$

In this article, motivated by the work in [10]-[12], based on the asymptotic regularity in [9], we construct a finite dimensional exponential attractor of (1.1) only under the conditions (1.2) and (1.3).

Our main result is

Theorem 1.1 Assume $f \in \mathcal{C}^{1}(\mathbb{R})$ and satisfies (1.2)-(1.3), $g \in H^{-1}(\Omega)$. Then the semigroup $\{S(t)\}_{t \geq 0}$ associated with problem (1.1) has an exponential attractor $\mathcal{E}$ in $H_{0}^{1}(\Omega)$.

Remark 1.1 If $\mathcal{A}$ is a global attractor of (1.1) in $H_{0}^{1}(\Omega)$, we know that $\mathcal{A} \subset \mathcal{E}$, then Theorem 1.1 implies that fractal dimension of the global attractor $\mathcal{A}$ is finite.

\section{Notations and Preliminaries}

In this section, for convenience, we introduce some notations about the functions space which will be used later throughout this article.

- $A=-\Delta$ with domain $D(A)=H^{2} \cap H_{0}^{1}$, and consider the family of Hilbert space $D\left(A^{s / 2}\right), s \in \mathbb{R}$ with the 
standard inner products and norms, respectively,

$$
\langle\cdot \cdot\rangle_{D\left(A^{s / 2}\right)}=\left\langle A^{s / 2} \cdot A^{s / 2} \cdot\right\rangle \text { and }\|\cdot\|_{D\left(A^{s / 2}\right)}=\left\|A^{s / 2} \cdot\right\| \text {. }
$$

Especially, $\langle\cdot, \cdot\rangle$ and $\|\cdot\|$ means the $L^{2}(\Omega)$ inner product and norm, respectively.

- $\mathcal{H}^{s}=D\left(A^{\frac{1+s}{2}}\right), s \in[0,1]$ with the usual norm $\|u\|_{\mathcal{H}^{s}}^{2}=\left\|A^{\frac{1+s}{2}} u\right\|^{2}$. Especially, we denote $\mathcal{H}=\mathcal{H}^{0}=H_{0}^{1}$ and $\|\cdot\|_{\mathcal{H}}=\|\cdot\|_{H_{0}^{1}} \cdot$

- $Q(\cdot), Q_{i}(\cdot):[0, \infty) \mapsto[0, \infty), i=1,2, \cdots$ are continuous increasing functions.

- $C, C_{i}$ denote the general positive constants, $i=1,2, \cdots$, which will be different from line to line.

We also need the following the transitivity property of exponential attraction, e.g., see [[12], Theorem 5.1]:

Lemma 2.1 ([13]) Let $\mathcal{K}_{1}, \mathcal{K}_{2}, \mathcal{K}_{3}$ be subsets of $\mathcal{H}$ such that

$$
\operatorname{dist}_{\mathcal{H}}\left(S(t) \mathcal{K}_{1}, \mathcal{K}_{2}\right) \leq L_{1} \mathrm{e}^{-\mu_{1} t}, \operatorname{dist}_{\mathcal{H}}\left(S(t) \mathcal{K}_{2}, \mathcal{K}_{3}\right) \leq L_{2} \mathrm{e}^{-\mu_{2} t},
$$

for some $\mu_{1}, \mu_{2}>0$ and $L_{1}, L_{2} \geq 0$. Assume also that for all $z_{1}, z_{2} \in \bigcup_{t \geq 0} S(t) \mathcal{K}_{j}(j=1,2,3)$ there holds

$$
\left\|S(t) z_{1}-S(t) z_{2}\right\|_{\mathcal{H}} \leq L_{0} \mathrm{e}^{-\mu_{0} t}\left\|z_{1}-z_{1}\right\|_{\mathcal{H}},
$$

for some $\mu_{0} \geq 0$ and $L_{0} \geq 0$. Then it follows that

$$
\operatorname{dist}_{\mathcal{H}}\left(S(t) \mathcal{K}_{1}, \mathcal{K}_{3}\right) \leq L \mathrm{e}^{-\mu t},
$$

where $\mu=\frac{\mu_{1} \mu_{2}}{\mu_{0}+\mu_{1}+\mu_{2}}$ and $L=L_{0} L_{1}+L_{2}$.

\section{Exponential Attractor}

In this subsection, based on the asymptotic regularity obtained in [9], we will construct an exponential attractor by the methods and techniques devised in [10]-[12]. We first need the following Lemmas:

Lemma 3.1 ([7]) Let $f \in \mathcal{C}^{1}(\mathbb{R})$ satisfies (1.2)-(1.3) and $g \in H^{-1}(\Omega)$. Then for any $u_{0} \in \mathcal{H}$ and any $T>0$, there is a unique solution $u$ of (1.1) such that

$$
u \in \mathcal{C}([0, T] ; \mathcal{H}) \cap L^{\infty}\left(0, \infty ; \mathcal{H}^{1}\right), u_{t} \in L^{2}(0, T ; \mathcal{H})
$$

Moreover,the solution continuously depends on the initial data in $\mathcal{H}$.

In the remainder of this section, we denote by $\{S(t)\}_{t \geq 0}$ the semigroup associated with the solutions of (1.1)-(1.3).

Lemma 3.2 ([7]) Under conditions of above Lemma, There is a positive constant $\rho$ such that for any bounded subset $B \subset \mathcal{H}$, there exists $T=T(B)$ such that

$$
\left\|S(t) u_{0}\right\|_{\mathcal{H}}(=\|\nabla u\|) \leq \rho \text {, for all } t \geq T \text { and } u_{0} \in B .
$$

From this Lemma, we know that the semigroup of operators $\{S(t)\}_{t \geq 0}$ generalized by (1.1) possesses a bounded absorbing set $\mathcal{B}_{0}$ in $\mathcal{H}$.

Lemma 3.3 Under conditions of Lemma3.1, and $u(t), v(t)$ be two solutions of (1.1) with $u(0)=u_{0}, v(0)=v_{0}$, respectively, it follows that

$$
\|u(t)-v(t)\|_{\mathcal{H}}^{2} \leq\|u(0)-v(0)\|_{\mathcal{H}}^{2} \mathrm{e}^{\mathrm{Ct}} \text {, for all } t \geq 0 .
$$

Proof Let $w=u-v$ satisfies the following equation

$$
w_{t}+A w_{t}+A w+f(u)-f(v)=0 .
$$

Taking the scalar product of (3.3) with $w$, we find,

$$
\frac{1}{2} \frac{\mathrm{d}}{\mathrm{d} t}\left(\|w\|^{2}+\|\nabla w\|^{2}\right)+\|\nabla w\|^{2}=-\langle f(u)-f(v), w\rangle .
$$


From the condition (1.2), by using the Hölder inequality, and noting the embedding $H_{0}^{1} \hookrightarrow L^{\frac{2 N}{N-2}}$, we have

$$
|\langle f(u)-f(v), w\rangle| \leq C\left(1+\|\nabla u\| \frac{4}{N-2}+\|\nabla v\|^{\frac{4}{N-2}}\right)\|\nabla w\|^{2}, \forall u, v \in \mathcal{H} .
$$

And then, by means of (3.1), we obtain

$$
|\langle f(u)-f(v), w\rangle| \leq C\|\nabla w\|^{2}, \forall u, v \in \mathcal{H} .
$$

So, combining with Equation (3.4), (3.5), we get

$$
\frac{\mathrm{d}}{\mathrm{d} t}\left(\|w\|^{2}+\|\nabla w\|^{2}\right) \leq C\left(\|w\|^{2}+\|\nabla w\|^{2}\right),
$$

then using the Gronwall lemma to above inequality, we can conclude our lemma immediately.

Lemma 3.4 ([9]) Let $f \in \mathcal{C}^{1}(\mathbb{R})$ and satisfies (1.2), (1.3), $g \in H^{-1}$. Then, for any

$\sigma \in\left[0, \min \left\{1, \frac{N}{2}-1\right\}\right)$, there exists a subset $\mathcal{B}_{\sigma}$, a positive constant $\eta$ and a monotone increasing function $Q_{\sigma}(\cdot)$ such that for any bounded set $B \subset H_{0}^{1}$,

$$
\operatorname{dist}_{H_{0}^{1}}\left(S(t) B, \mathcal{B}_{\sigma}\right) \leq Q_{\sigma}\left(\|B\|_{H_{0}^{1}}\right) \mathrm{e}^{-\eta t} \text {, for all } t \geq 0,
$$

where $\mathcal{B}_{\sigma}$ and $Q_{\sigma}(\cdot)$ depend on $\sigma$ but $\eta$ is independent of $\sigma ; \mathcal{B}_{\sigma}$ satisfying

$$
\mathcal{B}_{\sigma}=\left\{z \in H_{0}^{1}:\|z-\phi(x)\|_{H^{1+\sigma}} \leq \Lambda_{\sigma}<\infty\right\}
$$

for some positive constant $\Lambda_{\sigma}$; And $\phi(x)$ is the unique solution of the following elliptic equation

$$
\left\{\begin{array}{l}
-\Delta \phi+f(\phi)+(l+1) \phi=g(x)-g^{v}, \text { in } \Omega, \\
\left.\phi\right|_{\partial \Omega}=0,
\end{array}\right.
$$

where the constant $l>0, g^{v} \in L^{2}(\Omega)$ such that $\left\|g-g^{v}\right\|_{H^{-1}}<\zeta \ll 1$. Furthermore, we know that the solution $\phi(x)$ only belongs to $H_{0}^{1}(\Omega)$ when $f$ satisfies (1.2)-(1.3).

Lemma 3.5 ([9]) Under the assumption of Lemma 3.4, for any bounded subset $B_{1} \subset \mathcal{H}^{\sigma}$, if the initial data $u_{0} \in \phi(x)+B_{1}$, then the solution $u(t)$ of (1.1) has the following estimates similar to (3.7) in Lemma 3.4, more precisely, we have

$$
\left\|S(t) u_{0}-\phi(x)\right\|_{\mathcal{H}^{\sigma}}^{2}=\|u(t)-\phi(x)\|_{\mathcal{H}^{\sigma}}^{2} \leq M_{B_{1}}, \forall t \geq 0, u_{0} \in \phi(x)+B_{1},
$$

where the constant $M_{B_{1}}$ depends only on $\sigma$ and the $\mathcal{H}^{\sigma}$-bound of $B_{1}$.

Lemma 3.6 There exists $C>0$ such that

$$
\int_{t}^{t+1}\left\|u_{t}(\tau)\right\|_{\mathcal{H}} \mathrm{d} \tau \leq C, \text { for all } t \geq 0 .
$$

Proof For the solution $u(x, t)$ of (1.1), we now decompose $u(x, t)$ as follows

$$
u(x, t)=\phi(x)+w(x, t),
$$

where $\phi(x)$ is a fixed solution of (3.8), and $w(x, t)$ satisfies the following equation :

$$
\left\{\begin{array}{l}
w_{t}-\Delta w_{t}-\Delta w+f(u)-f(\phi)-(l+1) \phi=g^{v}, \\
w(x, 0)=u_{0}-\phi .
\end{array}\right.
$$

At the same time, noticing the embedding $H^{\sigma} \longmapsto H$, and from Lemma 3.5 we yield

$$
\|w(t)\|_{\mathcal{H}} \leq\|w(t)\|_{\mathcal{H}^{\sigma}} \leq M_{B_{1}} .
$$


Taking the inner product of (3.12) with $w_{t}$, we get

$$
\left\|w_{t}\right\|^{2}+\left\|\nabla w_{t}\right\|^{2}+\frac{1}{2} \frac{\mathrm{d}}{\mathrm{d} t}\|\nabla w\|^{2}=-\left\langle f(u)-f(\phi), w_{t}\right\rangle+\left\langle(l+1) \phi, w_{t}\right\rangle+\left\langle g^{v}, w_{t}\right\rangle .
$$

By means of (3.1) and (3.13) and together with Hö lder, Young inequalities, it follows that

$$
\begin{gathered}
\left|-\left\langle f(u)-f(\phi), w_{t}\right\rangle\right| \leq C \int_{\Omega}\left(1+|u| \frac{4}{N-2}+|\phi| \frac{4}{N-2}\right)|w|\left|w_{t}\right| \leq C\left(1+\|u\|_{L^{\frac{2 N}{N-2}}}^{\frac{4}{N-2}}+\|\phi\|_{\frac{2 N}{L^{N-2}}}^{\frac{4}{N-2}}\right) \cdot\|w\|_{L^{N-2}}^{\frac{2 N}{N-2}} \cdot\left\|w_{t}\right\|_{L^{\frac{2 N}{N-2}}} \\
\leq C\left(1+\|\nabla u\| \frac{4}{N-2}+\|\nabla \phi\| \frac{4}{N-2}\right)\|w\|_{\mathcal{H}}\left\|\nabla w_{t}\right\| \leq C+\frac{1}{3}\left\|\nabla w_{t}\right\|^{2} ; \\
\left\langle(l+1) \phi, w_{t}\right\rangle \leq C(l+1)\|\nabla \phi\|^{2}+\frac{1}{3}\left\|\nabla w_{t}\right\|^{2} ; \\
\left\langle g^{v}, w_{t}\right\rangle \leq C\left\|g^{v}\right\|+\frac{1}{3}\left\|\nabla w_{t}\right\|^{2} .
\end{gathered}
$$

Thus, combining with (3.14), there holds

$$
\left\|w_{t}\right\|^{2}+\frac{1}{2} \frac{\mathrm{d}}{\mathrm{d} t}\|\nabla w\|^{2} \leq C .
$$

Integrating the above inequality on $(t, t+1)$ and noting $u_{t}=w_{t}$, the proof completes.

Next, we will prepared for constructing an exponential attractor of $\{S(t)\}_{t \geq 0}$ in $\mathcal{H}$ by applying the abstract results devised in [10]-[12] [14].

Firstly, for each fixed $\sigma \in\left[0, \min \left\{1, \frac{N}{2}-1\right\}\right)$, we define

$$
\mathcal{X}={\overline{\bigcup_{\tau \geq 1} S(\tau) \mathcal{B}_{\sigma}}}^{\mathcal{H}},
$$

where $\mathcal{B}_{\sigma}$ is the set obtained in Lemma 3.4. Then, from Lemma 3.5 we know that

$$
\|\mathcal{X}-\phi(x)\|_{\mathcal{H}^{\sigma}}<\infty \text {. }
$$

Secondly, let us establish some properties of this set.

- $\mathcal{X}$ is a compact set in $\mathcal{H}=H_{0}^{1}$, due to Lemma 3.4.

- $\mathcal{X}$ is positive invariant. In fact, from the continuity of $S(t)$, we have

$$
S(t) \mathcal{X} \subset{\overline{\bigcup_{\tau \geq 1} S(t+\tau) \mathcal{B}_{\sigma}}}^{\mathcal{H}} \subset \mathcal{X} .
$$

- There holds

$$
\operatorname{dist}_{\mathcal{H}}\left(S(t) \mathcal{B}_{0}, \mathcal{X}\right) \leq Q_{\sigma}\left(\|B\|_{H_{0}^{1}}\right) \mathrm{e}^{-\eta t} \text {, for all } t \geq 0
$$

Indeed, it is apparent that

$$
\operatorname{dist}_{\mathcal{H}}\left(S(t) \mathcal{B}_{\sigma}, \mathcal{X}\right)=0, \forall t \geq 1
$$

Hence, (3.19) follows from Lemma 2.1.

- There is $C>0$ such that

$$
\int_{t}^{t+1}\left\|u_{t}(\tau)\right\|_{\mathcal{H}} \mathrm{d} \tau \leq C, u_{0} \in \mathcal{X} \text {, for all } t \geq 0 .
$$

This is a direct consequence of Lemma 3.6.

Therefore such a set $\mathcal{X}$ is a promising candidate for our purpose.

Finally, we need the following two lemmas.

Lemma 3.7 For every $T>0$, the mapping $\left(t, u_{0}\right) \mapsto S(t) u_{0}$ is Lipschitz continuous on $[0, T] \times \mathcal{X}$. 
Proof For $u_{1}, u_{2} \in \mathcal{X}$ and $t_{1}, t_{2} \in[0, T]$ we have

$$
\left\|S\left(t_{1}\right) u_{1}-S\left(t_{2}\right) u_{2}\right\|_{\mathcal{H}} \leq\left\|S\left(t_{1}\right) u_{1}-S\left(t_{1}\right) u_{2}\right\|_{\mathcal{H}}+\left\|S\left(t_{1}\right) u_{2}-S\left(t_{2}\right) u_{2}\right\|_{\mathcal{H}}
$$

The first term of the above inequality is handled by estimate (3.2). Concerning the second one,

$$
\left\|S\left(t_{1}\right) u_{2}-S\left(t_{2}\right) u_{2}\right\|_{\mathcal{H}}=\left\|u\left(t_{1}\right)-\left.u\left(t_{2}\right)\right|_{\mathcal{H}} \leq\left|\int_{t_{1}}^{t_{2}}\right|\left|u_{t}(y) \|_{\mathcal{H}} \mathrm{d} y\right| \leq C\left|t_{1}-t_{2}\right| .\right.
$$

Hence, there exists a constant $L=L(t)>0$, such that

$$
\left\|S\left(t_{1}\right) u_{1}-S\left(t_{2}\right) u_{2}\right\|_{\mathcal{H}} \leq L\left[\left|t_{1}-t_{2}\right|+\left\|u_{1}-u_{2}\right\|_{\mathcal{H}}\right] .
$$

On the other hands, for each initial data $\xi_{u}(0) \in \mathcal{X}$, we can decompose the solution $u(t)$ of (1.1) as

$$
u(t)=S_{1}(t) \xi_{u}(0)+S_{2}(t) \xi_{u}(0),
$$

where $\bar{v}(t)=S_{1}(t) \xi_{u}(0)$ and $\bar{w}(t)=S_{2}(t) \xi_{u}(0)$ solve the following equations respectively:

$$
\left\{\begin{array}{l}
\bar{v}_{t}-\Delta \bar{v}_{t}-\Delta \bar{v}=g(x), \\
\bar{v}(0)=\xi_{u}(0) \\
\left.\bar{v}\right|_{\partial \Omega}=0
\end{array}\right.
$$

and

$$
\left\{\begin{array}{l}
\bar{w}_{t}-\Delta \bar{w}_{t}-\Delta \bar{w}+f(u)=0, \\
\bar{w}(0)=0 \\
\left.\bar{w}\right|_{\partial \Omega}=0 .
\end{array}\right.
$$

Therefore, we will have the following lemma:

Lemma 3.8 The following two estimates hold:

$$
\left\|S_{1}\left(t^{*}\right) \xi_{u_{1}}(0)-S_{1}\left(t^{*}\right) \xi_{u_{2}}(0)\right\|_{\mathcal{H}} \leq \frac{1}{4}\left\|\xi_{u_{1}}(0)-\xi_{u_{2}}(0)\right\|_{\mathcal{H}} \text {, for all } \xi_{u_{1}}(0), \xi_{u_{2}}(0) \in \mathcal{X},
$$

and

$$
\left\|S_{2}\left(t^{*}\right) \xi_{u_{1}}(0)-S_{2}\left(t^{*}\right) \xi_{u_{2}}(0)\right\|_{\mathcal{H}^{\sigma}} \leq K\left\|\xi_{u_{1}}(0)-\xi_{u_{2}}(0)\right\|_{\mathcal{H}} \text {, for all } \xi_{u_{1}}(0), \xi_{u_{2}}(0) \in \mathcal{X},
$$

where the constant $K$ depends only on $t^{*}$ and $\|\mathcal{X}-\phi(x)\|_{\mathcal{H}^{\sigma}}$.

Proof Given two solutions $u_{1}, u_{2}$ of Equation (1.1) origination from $\xi_{u_{1}}(0), \xi_{u_{2}}(0) \in \mathcal{X}$, respectively.

Set

$$
\bar{u}=u_{1}-u_{2}=S(t) \xi_{u_{1}}(0)-S(t) \xi_{u_{2}}(0) \triangleq \overline{\bar{v}}+\overline{\bar{w}} .
$$

where $\overline{\bar{v}}(t)$ and $\overline{\bar{w}}(t)$ solve the following equations respectively:

$$
\left\{\begin{array}{l}
\overline{\bar{v}}_{t}-\Delta \overline{\bar{v}}_{t}-\Delta \overline{\bar{v}}=0, \\
\overline{\bar{v}}(0)=\xi_{u_{1}}(0)-\xi_{u_{2}}(0),
\end{array}\right.
$$

and

$$
\left\{\begin{array}{l}
\overline{\bar{w}}_{t}-\Delta \overline{\bar{w}}_{t}-\Delta \overline{\bar{w}}+f\left(u_{1}\right)-f\left(u_{2}\right)=0, \\
\overline{\bar{w}}(0)=0 .
\end{array}\right.
$$

It is apparent that $\overline{\bar{v}}(t)=S_{1}(t) \xi_{u_{1}}(0)-S_{1}(t) \xi_{u_{2}}(0)$ and $\overline{\bar{w}}(t)=S_{2}(t) \xi_{u_{1}}(0)-S_{2}(t) \xi_{u_{2}}(0)$.

Taking the product of (3.30) with $\overline{\bar{v}}$ in $L^{2}(\Omega)$, we get

$$
\frac{\mathrm{d}}{\mathrm{d} t}\left(\|\overline{\bar{v}}(t)\|^{2}+\|\nabla \overline{\bar{v}}(t)\|^{2}\right)+C_{1}\left(\|\overline{\bar{v}}(t)\|^{2}+\|\nabla \overline{\bar{v}}(t)\|^{2}\right) \leq 0
$$


So

$$
\|\overline{\bar{v}}(t)\|^{2}+\|\nabla \overline{\bar{v}}(t)\|^{2} \leq C\left(\|\overline{\bar{v}}(0)\|^{2}+\|\nabla \overline{\bar{v}}(0)\|^{2}\right) \mathrm{e}^{-C_{1} t}
$$

Hence, setting

$$
t^{*}=\frac{1}{C_{1}} \log 4 C
$$

we have

$$
\left\|S_{1}\left(t^{*}\right) \xi_{u_{1}}(0)-S_{1}\left(t^{*}\right) \xi_{u_{2}}(0)\right\|_{\mathcal{H}} \leq \frac{1}{4}\left\|\xi_{u_{1}}(0)-\xi_{u_{2}}(0)\right\|_{\mathcal{H}} \text {, for all } \xi_{u_{1}}(0), \xi_{u_{2}}(0) \in \mathcal{X}
$$

So, we obtain the result (3.28).

On the other hands, taking the product of (3.31) with $A^{\sigma} \overline{\bar{w}}$ in $L^{2}(\Omega)$, we ge

$$
\frac{1}{2} \frac{\mathrm{d}}{\mathrm{d} t}\left(\left\|A^{\frac{\sigma}{2}} \overline{\bar{w}}\right\|^{2}+\left\|A^{\frac{1+\sigma}{2}} \overline{\bar{w}}\right\|^{2}\right)+\left\|A^{\frac{1+\sigma}{2}}\right\|^{2} \|^{2}=-\left\langle f\left(u_{1}\right)-f\left(u_{2}\right), A^{\sigma} \overline{\bar{w}}\right\rangle .
$$

Since $\sigma \in\left[0, \min \left\{1, \frac{N}{2}-1\right\}\right)$, we have $\frac{2 N}{4}<\frac{2 N}{N-2+(6-N) \sigma} \leq \frac{2 N}{N-2}$.

So, from (1.2) and using $\mathrm{H} \ddot{o}$ lder inequality, we have

$$
\begin{aligned}
& -\left\langle f\left(u_{1}\right)-f\left(u_{2}\right), A^{\sigma} \overline{\bar{w}}\right\rangle \leq \int_{\Omega}\left(1+\left|u_{1} \frac{4}{N-2}+\right| u_{2} \mid \frac{4}{N-2}\right)|\bar{u}|\left|A^{\sigma} \overline{\bar{w}}\right| \\
& \leq C_{2}\left(1+\left\|u_{1}\right\|_{L^{\frac{2 N}{N-2-2 \sigma}}}^{\frac{4}{N-2}}+\left\|u_{2}\right\|_{L^{\frac{2 N}{N-2-2 \sigma}}}^{\frac{4}{N-2}}\right) \cdot\|\bar{u}\|_{L^{N-2+(6-N) \sigma}} \frac{2 N}{\bar{N}} \cdot\left\|A^{\sigma} \overline{\bar{w}}\right\|_{L^{\frac{2 N}{N-2+2 \sigma}}} \cdot \mid \Omega \frac{4 \sigma}{\frac{4 \sigma}{N(N-2)}}-\frac{(8-N) \sigma}{2 N} \\
& \leq C_{2} C(|\Omega|, \sigma)\left(1+\left\|\left.u_{1}\right|_{\mathcal{H}^{\sigma}} ^{\frac{4}{N-2}}+\right\| u_{2} \|_{\mathcal{H}^{\sigma}}^{\frac{4}{N-2}}\right)\|\bar{u}\|_{\mathcal{H}}\left\|A^{\frac{1+\sigma}{2}} \overline{\bar{w}}\right\| \leq C_{2} C(|\Omega|, \sigma)\|\bar{u}\|_{\mathcal{H}}^{2}+\left\|A^{\frac{1+\sigma}{2}} \overline{\bar{w}}\right\|^{2},
\end{aligned}
$$

where the constant $C_{2}$ comes from the embedding $\mathcal{H}=H_{0}^{1}(\Omega) \hookrightarrow L^{\frac{2 N}{N-2}}(\Omega), D\left(A^{\frac{1+\sigma}{2}}\right) \hookrightarrow L^{\frac{2 N}{N-2-2 \sigma}}(\Omega)$, $D\left(A^{\frac{1-\sigma}{2}}\right) \hookrightarrow L^{\frac{2 N}{N-2+2 \sigma}}(\Omega)$.

From Lemma 3.3, we obtain the inequality

$$
\frac{\mathrm{d}}{\mathrm{d} t}\left(\left\|A^{\frac{\sigma}{2}} \overline{\bar{w}}\right\|^{2}+\left\|A^{\frac{1+\sigma}{2}} \overline{\bar{w}}\right\|^{2}\right) \leq C \mathrm{e}^{C t}\left\|u_{1}-u_{2}\right\|^{2} .
$$

and an integration on $\left(0, t^{*}\right)$, we can get the estimate (3.29).

Proof of Theorem 1.1 Applying the abstract results devised in [10]-[12], from Lemma 3.7 and Lemma 3.8, we can prove the existence of an exponential attractor $\mathcal{E}$ for $\{S(t)\}_{t \geq 0}$ in $\mathcal{H}\left(=H_{0}^{1}\right)$ immediately.

Remark 3.9 As a direct consequence of Theorem 1.1 and the a priori estimates given in [[9], Lemma 3.5] and Lemma 3.8, we decompose $\mathcal{E}$ as $\mathcal{E}=\phi(x)+\mathcal{E}^{\prime}$, where $\mathcal{E}^{\prime}$ is bounded in $\mathcal{H}^{\sigma}$ for any $\sigma \in\left[0, \min \left\{1, \frac{N}{2}-1\right\}\right)$ and $\phi(x)$ is the unique solution of (3.8).

\section{Acknowledgements}

The authors thank the referee for his/her comments and suggestions, which have improved the original version of this article essentially. This work was partly supported by the NSFC $(11061030,11101334)$ and the NSF of 
Gansu Province(1107RJZA223), in part by the Fundamental Research Funds for the Gansu Universities.

\section{References}

[1] Aifantis, E.C. (1980) On the Problem of Diffusion in Solids. Acta Mechanica, 37, 265-296. http://dx.doi.org/10.1007/BF01202949

[2] Kuttler, K. and Aifantis, E. (1988) Quasilinear Evolution Equations in Nonclassical Diffusion. SIAM Journal on Applied Mathematics, 19, 110-120. http://dx.doi.org/10.1137/0519008

[3] Peter, J.G. and Gurtin, M.E. (1968) On the Theory of Heat Condition Involving Two Temperatures. Zeitschrift für angewandte Mathematik und Physik ZAMP, 19, 614-627. http://dx.doi.org/10.1007/BF01594969

[4] Xiao, Y. (2002) Attractors for a Nonclassical Diffusion. Acta Mathematicae Applicatae Sinica, 18, 273-276. http://dx.doi.org/10.1007/s102550200026

[5] Wang, S., Li, D. and Zhong, C. (2006) On the Dynamics of a Class of Nonclassical Parabolic Equation. Journal of Mathematical Analysis and Applications, 317, 565-582. http://dx.doi.org/10.1016/j.jmaa.2005.06.094

[6] Sun, C., Wang, S. and Zhong, C. (2007) Global Attractors for a Nonclassical Diffusion Equation. Acta Mathematicae Applicatae Sinica, 23, 1271-1280.

[7] Sun, C. and Yang, M. (2008) Dynamics of the Nonclassical Diffusion Equations. Asymptotic Analysis, 59, 51-81.

[8] Sun, C. (2009) Asympotic Regularity for Some Dissipative Equations. Journal of Differential Equations, 248, $342-362$. http://dx.doi.org/10.1016/j.jde.2009.08.007

[9] Wu, H. and Zhang, Z. (2011) Asymptotic Regularity for the Nonclassical Diffusion Equation with Lower Regular Foring Term. Dynamical Systems, 26, 391-400. http://dx.doi.org/10.1080/14689367.2011.562185

[10] Sun, C., Yang, L. and Duan, J. (2011) Asymptotic Behavior for a Semilinear Second Order Evolution Equation. Transactions of the American Mathematical Society, 363, 6085-6109. http://dx.doi.org/10.1090/S0002-9947-2011-05373-0

[11] Efendiev, M., Miranville, A. and Zelik, S.V. (2005) Exponential Attractors and Finite-Dimensional Reduction of NonAutonomous Dynamical Systems. Proceedings of the Royal Society of Edinburgh: Section A Mathematics, 135, 703730.

[12] Miranville, A. and Zelik, S.V. (2008) Attracts for Dissipative Partial Different Equations in Bounded and Unbounded Domains, In: Dafermos, C.M. and Pokorny, M., Eds., Evolutionary Equations, Elsevier, Amesterdam, 103.

[13] Fabrie, P., Galusinski, C., Miranville, A. and Zelik, S. (2004) Uniform Exponential Attractors for a Singularly Perturbed Damped Wave Equation. Discrete and Continuous Dynamical Systems, 10, 211-238. http://dx.doi.org/10.3934/dcds.2004.10.211

[14] Pata, V. and Squassina, M. (2005) On the Strongly Damped Wave Equation. Communications in Mathematical Physics, 253, 511-533. http://dx.doi.org/10.1007/s00220-004-1233-1 